\title{
Die Neue DZA!
}

Wir begrüßen die Leser der DZA im Neuen Jahr mit der 1. Ausgabe 2018, verlegt und editiert vom Springer Medizin Verlag.

Seit der einvernehmlichen Auflösung des Vertrages mit Elsevier 2016 und dem Dank an liebgewordene Mitarbeiter haben wir uns auf die Suche nach einem neuen Verlag begeben, einem Verlag dem wir „unsere“ DZA anvertrauen können. Es fanden Orientierungsgespräche mit den meisten großen deutschen, sowie einigen mittleren und kleineren Medizinverlagen statt.

Ebenso wurde die Idee eines Eigenverlages verfolgt, geprüft und dann aus verschiedenen Gründen wieder verworfen. Auf der Basis der Gespräche mit externen Beratern, sowie den Verlagen und unseren Erfahrungen wurde eine Ausschreibung gestaltet und verbreitet. Nach Vorab-Prüfung wurden dann 6 Verlage zur Anhörung nach München eingeladen

Nach Konsensfindung auf der Basis definierter Bewertungskriterien und nach intensiven Diskussionen haben sich die Herausgebergesellschaften entschlossen, Verhandlungen mit dem Springer Medizin Verlag aufzunehmen. Dieser wurde 1842 durch Julius Springer gegründet ist heute Teil des global aktiven SPRINGER NATURE Verlages.

Es folgten vier Monate Verhandlungen, die dann in einem für beide Seiten guten Ergebnis endeten. Grundlage ist und bleibt das gegenseitige Vertrauen, so wurde dann auch im Oktober 2017 die erste Redaktionskonferenz einberufen, obwohl Vertragsdetails noch nicht in paragraphensichere Form eingeflossen waren. Nun sind jedoch die Verträge unterschrieben.

Diese erste Redaktionskonferenz war dann der Beginn zu einer Neuaufstellung der DZA. Vorab wurde ein Aufruf zur redaktionellen Mitarbeit veröffentlicht, auf den sich doch einige im Schreiben erfahrene und interessierte Kolleginnen und Kollegen meldeten. Viele der bewährten Mitarbeiter sind weiterhin dabei, sodass sich nun eine engagierte Gruppe von Redakteuren und Mitarbeitern gefunden hat, die viel versprechen lässt.

Bei der Sitzung wurden neue Rubriken entwickelt, jeweils Verantwortliche und Mitarbeiter festgelegt, und interessante Themenbereiche und Schwerpunkte ausgearbeitet.

Um dem ganzen Spektrum der DZA formal auch Ausdruck zu geben, wurden die drei Bereiche „Wissenschaft", „Praxis“ und "Gesundheitswesen/Soziales“ als Hauptrubriken festgelegt und weiter unterteilt. Sie finden die Rubriken, ihre Verantwortlichen und die entsprechenden Redakteure und Mitarbeiter auf den ersten Seiten jeder Ausgabe aufgelistet. Die Zuständigkeiten wurden zunächst für ein Jahr zugeteilt, um eine Durchlässigkeit und Entwicklungen zuzulassen.

Mit großer Freude sehen wir als Herausgeber, dass die Chefredaktion weiterhin in den bewährten Händen von Dr. Dr. Tom Ots bleibt. Seine Erfahrungen und Kompetenz wird die Konti-

Deutsche Zeitschrift für Akupunktur

$2018 \cdot 61$ (1): 1

https://doi.org/10.1007/s42212-018-0028-9

๑) Springer Medizin Verlag GmbH, ein Teil von Springer Nature 2018

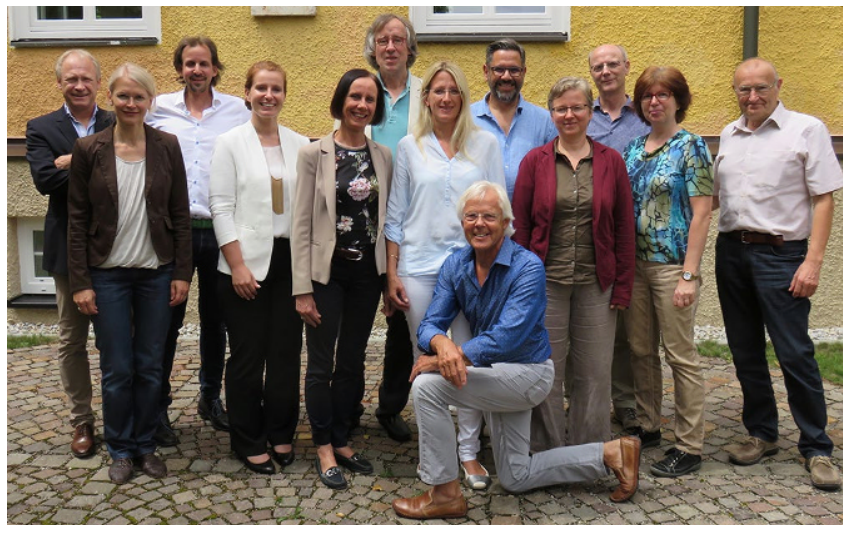

Herausgeber, Frau Kretz und Frau Stratmann vom Springer Verlag, sowie alte und neue Redakteurlnnen

nuität der DZA sichern. Ihm gilt auch unser Dank für die intensive Mitarbeit beim Übergang von Elsevier zu Springer und die Federführung bei der Zusammenstellung der neuen Redaktion.

Unser Dank gebührt besonders Herrn Dr. med. Paul Herrmann, Director Journals \& ePublishing und Frau Monika Kretz, Head of Journals \& ePublishing, Springer Medizin Verlag GmbH für die offenen und konstruktiven Verhandlungen.

Managing Editor Verena Stratmann wird die DZA in Zukunft im Alltag begleiten. Die bisherige, wenn auch noch kurze Zusammenarbeit bestätigt unser Vertrauen und gibt uns Sicherheit für ein optimales Handling der Redaktionsprozesse.

So wünschen wir der neuen DZA einen guten Start, eine lebendige Leserschaft, erfolgreiche Wissensvermittlung und Erfahrungsaustausch, damit sie das bleibt, was sie seit Jahrzehnten ist:

Die größte und bedeutendste deutschsprachige Zeitschrift für Akupunktur, TCM und Related Techniques.

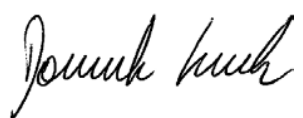

Priv. Doz. Dr. Dominik Irnich

Deutsche Ärztegesellschaft für Akupunktur e.V. (DÄGfA)

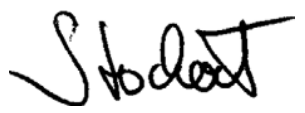

Dr. Karin Stockert

Österreichische Gesellschaft für Akupunktur e.V. (ÖGA)

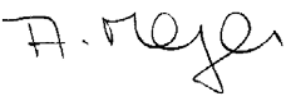

Dr. Anita Meyer

Schweizerische Ärztegesellschaft für Akupunktur Chinesische Medizin - Aurikulomedizin (SACAM)

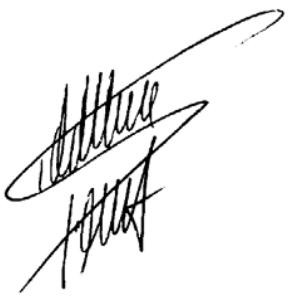

Dr. Siddhartha Popat M. A. Deutsche Gesellschaft für Akupunktur und Neuraltherapie e.V. (DGFAN)

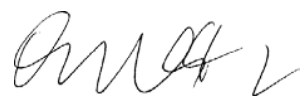

Dr. Elisabeth Preschitz Ärzte für Akupunktur e.V. (ÄfA) 